\title{
Comparison of Apolipoprotein B and Low Density Lipoprotein Cholesterol As Atherosclerotic Cardiovascular Risk Factors
}

Many large-scale studies have reported that current American Heart Association and American College of Cardiology guidelines overestimate Atherosclerotic Cardiovascular Disease (ASCVD) risk by $75 \%-150 \%$. People, who achieve low density lipoprotein (LDL) target with active statin therapy, still remain at a residual risk for cardiovascular disease and have high levels of apolipoprotein B (ApoB). The goal of this retrospective case-control study was to compare LDL and ApoB as predictors of ASCVD. Centers for Disease Control and Prevention's National Health and Examination Survey (NHANES) data from the 2013-2014 was the data source for this study. Around 5,768 subjects participated in the study and 1522 participants met the study criteria. Of these, $162($ Male $=93$, Female $=69)$ were cases (subjects with ASCVD) and 1,360 $($ Male $=629$, Female $=731)$ were controls (subjects without ASCVD). Chi-square test, correlation coefficient, and logistic regression were used to analyze data. P-value of $<0.05$ was considered to be significant. The study indicated that ApoB and LDL were strongly correlated (correlation coefficient $=0.8)$. Furthermore, LDL cholesterol was associated with ASCVD $(p$ $<0.05)$ but ApoB did not correlate with ASCVD ( $\mathrm{p}>0.05)$. Logistic regression results indicated that cases were 3 times more likely to have an increased LDL value but not likely to have an increased ApoB when compared to controls. The study concluded that LDL is an independent predictor of ASCVD and is a better marker than ApoB. 